\title{
How prepared are Myanmar's health care professionals in their response to gender-based violence?
}

\author{
Aye Nyein Ei and Montakarn Chuemchit \\ Chulalongkorn University College of Public Health Sciences, \\ Phathumwan, Bangkok, Thailand
}

\begin{abstract}
Purpose - Gender-based violence (GBV) has negative impact on the health and well-being of the survivors. The initial response can lessen the magnitude of the effect, and thus the health care sector's readiness to respond to GBV cases is important. This study aimed to explore the knowledge, attitude and its association to practice regarding GBV response among health care personnel.

Design/methodology/approach - A cross-sectional study was conducted in 48 public hospitals in Yangon, Myanmar, involving 398 participants including doctors and nurses, by a self-administered structured questionnaire.

Findings - Among 398 participants, most of the participants had moderate level of knowledge, attitude and supportive environmental factors. Only $12.8 \%$ had experienced GBV management. The respondents with a bachelor's degree were more likely to practice on health care management towards GBV cases than those who finished with a diploma degree. The medical officers and senior medical officers were more likely to practice than the junior nurses. The participants who had already attended the trainings had more practice than those who had not. Those who work in the regional hospitals were less likely to practice than those who work in the station-level hospital.

Originality/value - This paper explored the associated factors to health care personnel's practice of health care management towards GBV survivors in Myanmar which contains information about knowledge, attitude and supportive environmental factors. The study results can be used for a supportive data for health system strengthening the response of GBV cases in Myanmar.
\end{abstract}

Keywords Gender-based violence, Health care professionals' practices, Myanmar

Paper type Research paper

\section{Introduction}

Gender-based violence (GBV) against women has been defined as "any act that results in or is likely to result in physical, sexual, or psychological harm or suffering to women, including threats of such acts, coercion or arbitrary deprivation of liberty, whether occurring in public or private life" [1].

Globally, 35\% of women have experienced GBV [2]. In one study conducted among 600 participants from five townships in the Yangon Region, 19\% said they had faced intimate partner violence and 53\% mentioned that knew women who had been violated by

(C) Aye Nyein Ei and Montakarn Chuemchit. Published in the Journal of Health Research. Published by Emerald Publishing Limited. This article is published under the Creative Commons Attribution (CC BY 4.0) license. Anyone may reproduce, distribute, translate and create derivative works of this article (for both commercial and non-commercial purposes), subject to full attribution to the original publication and authors. The full terms of this license may be seen at http://creativecommons.org/licences/by/4.0/ legalcode

This research project was partially supported by the Graduate School Thesis Grant, Chulalongkorn University.

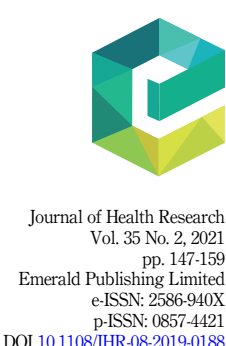

DOI 10.1108/JHR-08-2019-0188 
JHR

35,2

their family [3]. Another study carried out among 286 women in the Mandalay Region in 2005 reported that $69 \%$ of them had experienced domestic violence within the 12 months before the study. Among them, 69\% experienced psychological abuse and $27 \%$ experienced physical abuse [3].

The public health impact of GBV ranges from physical illness to social problems. Examples include physical illness such as injury, genital organ trauma, unwanted pregnancy and unsafe abortion; mental health problems such as anxiety, fear, shame and depression; and social problems such as victim-blaming and community stigma which can lead to disability, suicide or death [2]. GBV can also lead to other forms of socio-economic loss such as incomplete education that, in turn, impacts participation in the economic workforce whilst also having an additional impact on health care [4].

The impact of GBV on health may vary depending on the magnitude of the abuse, the personal data of the perpetrator (whether the abuser was a stranger or not), the magnitude of physical injuries, the support of the survivor's family and friends and other sectors as health and legal systems [5].

Among the various services for GBV survivors such as legal, social and counseling services, GBV victims might seek the support of a health care facility as their first source of support [6]. Survivors usually seek help at primary health care services, hospitals and other government and non-government sectors such as police, social welfare or women's support groups [6].

Health care services for survivors from GBV include (1) informed consent form, (2) history taking and physical examination, (3) forensic specimen collection, (4) prevention of pregnancy and diseases including STIs, HIV, hepatitis B, (5) psychosocial support, (6) follow-up visits and (7) referral [7, 8].

All health care personnel should obtain an informed consent form from clients that explains each step of all procedures that will be undertaken. Once this has been detailed and consent has been obtained, the general health history and reproductive health history should be taken along with a physical examination including the pelvic and anal region. If a forensic specimen is to be collected, it should be done during the physical examination to promote the survivor's comfort. The treatment process includes injuries treatment, STIs prevention, prevention of pregnancy, anti-tetanus, prevention of HIV and hepatitis B infection and psychosocial support and counseling. Follow-up visits should also be scheduled to assess the completion and side effects of treatment and the improvement in health. Accessibility, security, cleanliness, privacy and proper documentation are the features of these services [8].

The Ministry of Health and Sports in Myanmar has already developed a well-formed guideline for health sector response to GBV according to WHO guidelines. The book covers the background, initial support for the survivor of sexual or intimate partner violence, clinical care for a sexual violence survivor, psychosocial support and forensic specimen collection for legal support [9].

A report from EI (Educational Initiatives) and AJAR (Asia Justice and Rights) stated that various types of support services for GBV survivors in Myanmar exist in main townships and can be easily accessed by road. But the survivors from remote areas found difficulties in accessing these services due to lack of safe and secure transport services [3].

Therefore, if the health care personnel in various regions, including both urban and rural areas, are competent in how to manage the health problems of GBV survivors, the rate of awareness and action about access to health care services can be improved. Strengthening the health care personnel's competence and service delivery for survivors is the most basic form of change to ensure a better response to GBV [6]. One study in Yangon and Mandalay stated that the health care providers accept that GBV has certain health consequences and the mutual trust between the client and the provider can encourage the disclosure of private life. But some mentioned their lack of counseling capability concerning violence and some accepted intimate 
partner violence as a usual problem between husband and wife. From the view of clients, the relationship of trust with their health care providers, together with the providers' ability to support the survivors in choosing options and knowing their rights, enhanced their healthseeking behavior [10]. Another study in Malaysia found that health care providers in One Stop Crisis Centres (OSCC) had adequate knowledge on rape management, possessed positive attitudes towards rape victims and adhered to acceptable practices in the management of rape victims in OSCC [11]. A study in South Africa found the association between service providers' knowledge and attitude toward rape resulted in a more appropriate attitude toward rape [12]. However, a study in Korea revealed that nurses' awareness of suspected abuse cases was only $14.4 \%$, as they considered those cases were not severe because of lack of sufficient evidence and because they had a high workload [13], which is consistent with a study in Iran on barriers for providing services to the violent survivors. The study found that most survivors hid experience of violence from health professionals because of trust issues [14].

There are some studies conducted in Myanmar about barriers in seeking help by survivors from GBV. But there are limited studies on the factors that can determine the capacity of health professionals on health care for the GBV cases. Therefore, it is important to explore knowledge, attitude, supportive environmental factors and practice of health care personnel on health care management toward GBV survivors to be able to manage such cases. The resulting data will hopefully support health system strengthening the response of GBV cases. The authors hope that this paper also provides a better understanding of the situation of the health sector regarding GBV response in developing countries like Myanmar and helps to make some comparisons between them and the situation in developed countries.

\section{Methodology}

This was a cross-sectional study conducted among 398 health care personnel including doctors and nurses who worked in the emergency and out-patient departments (OPD) of public hospitals in the Yangon Region, Myanmar. This covered the total sample size needed, including a 10\% refusal rate calculated by Cochran's formula. The data were collected through self-administered questionnaires from late April to May 2019. The questionnaire contained five parts: (1) socio-demographic characteristics of the participants such as age, sex, working experience, training background related to GBV response, (2) knowledge on GBV response such as forms of GBV, physical examination for a GBV survivor, forensic specimen to be collected from a GBV survivor and referral methods, (3) attitude on GBV with the statements to be agreed, disagreed or remain neutral such as "health care personnel are responsible for exploring possible GBV cases," "domestic violence is a private matter" and "GBV can be experienced by all genders," (4) supportive environmental factors assessing the availability of the required infrastructure and medical supplies such as protocol, examination room, HIV rapid test kit, emergency contraception and referral contact for psychosocial support and (5) health care practices on GBV regarding the participants' experience of managing GBV cases within the previous two years. The questionnaire was built according to various literature reviews [11, 15-17]. The construct and content validity of the questionnaire was examined using the Item Objective Congruence (IOC score $=0.98$ ) index by three experts from the College of Public Health Sciences, Chulalongkorn University. The translated Myanmar questionnaire was checked by three professors from the Maternal and Child Health Department, Department of English Language and Department of Myanmar Language of University of Nursing, Yangon. A pilot test was also conducted among 40 health care personnel from other regions of Myanmar prior to the study. Multi-stage sampling was used in this study. The flow chart for the sampling procedure is shown in Figure 1.

In the first stage of the process, the Yangon Region was selected from 14 regions by purposive sampling as it had the highest reported GBV cases. Then, 15 townships were 


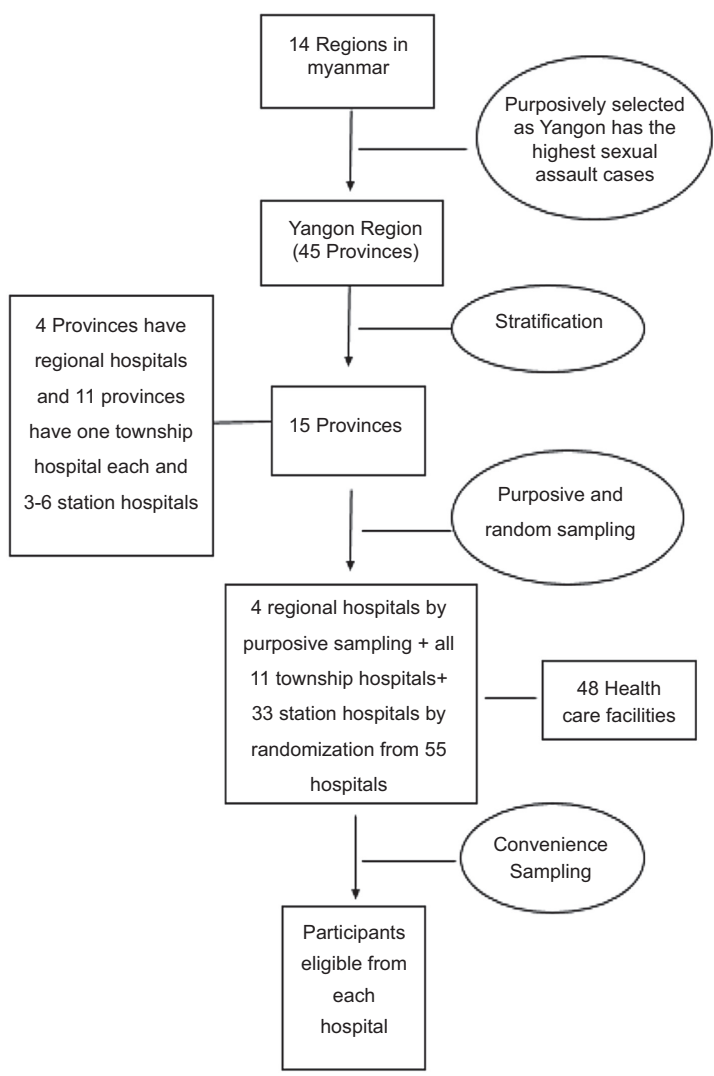

Figure 1.

Flow chart of sampling procedure

selected from 45 townships by stratified sampling to cover various levels of hospitals and to cover the needed sample size.

In the third stage, among the selected 15 townships, 4 townships had one regional-level hospital each, the remaining 11 townships had 1 township-level hospital, and 3-6 stationlevel hospitals from which every single township hospital was included and 3 station-level hospitals were randomly selected.

In the fourth stage, eligible participants were selected by convenience sampling. The estimated number of participants was about 20 from a regional hospital, 10 from township hospital and 7 from station hospital which is about $15 \%$ of the total health service providers working in each emergency and OPD department.

According to data collection protocol, permission for conducting the study was requested from the Yangon Regional Health Department (Ministry of Public Health) and from the Medical Superintendents of each hospital. With the official permission letter and the cooperation from the hospital administration, the research team could initiate communication and approach potential participants. After explaining the details of the study, the participants were requested to sign the written informed consent prior to participation in the study. Data collection was performed through self-administered questionnaires in the target population of health care personnel who were already familiar with the terms. Monitoring the completeness of data was performed by trained research assistants by 
checking the coverage of response to each question. All hospitals selected were included in the study as permission had been obtained from the regional administration.

Ethical approval was obtained from the Ethical Review Committee of Chulalongkorn University (COA No. 142/2019, Protocol No. 080.1/62).
Response to gender-based violence

\section{Results}

In this study, among 398 health care personnel (mean age $=35 \pm 8.265$ ), the majority of the participants were female $(86.2 \%)$ and most of them were senior nurses $(57 \%)$. Among them, $94 \%$ had the diploma degree and bachelor's degree equally. An equal proportion of respondents worked at each level of hospitals: station, township and regional; and the mean of the total working experience was 9.91 years.

This study assessed four different training types regarding GBV response. The study found that the vast majority, that is, $94.5 \%$ and $92.5 \%$, of the participants had never attended the GBV response training and the multidisciplinary teamwork training, respectively. Only $20 \%$ completed communication skills training, while 34.9\% finished STIs management training (Table 1).

\section{Knowledge, attitude and supportive environment factors toward health care for GBV cases}

The knowledge was assessed using nine questions. A correct answer was given 1 score and a 0 score for the wrong answer. The score varied from 0-45 points. Bloom's cut-off point (Bloom, 1968) was used to divide knowledge into three levels: (1) low level of knowledge $(<60 \%)$, (2) moderate level of knowledge (60-80\%) and (3) high level of knowledge $(>80 \%)$. Table 2 shows the level of knowledge of the participants toward GBV cases.

The mean score for attitudes was $18.65( \pm 2.313)$. Total attitude scores were classified into three levels, with a cut-off point of mean being (SD), 0-16 points indicated a negative attitude, $17-21$ points a neutral attitude and $22-24$ points a positive attitude. The study revealed that $17.1 \%$ of the participants had a negative attitude, $73.9 \%$ were neutral and only $9 \%$ of them had a positive attitude, Table 2 .

The supportive environmental factors contain facilities and infrastructures such as guidelines/protocols for GBV response, required documents, room for GBV counseling, HIV test kits and essential medications. Table 2 shows the level of the completeness of supportive environmental factors. The mean score was $4.29 \pm 1.643$. The level was high with a score of 7 , moderate if $4-6$ and low at $1-3$ (Table 2).

\section{Practices in health care management toward GBV cases}

For the practice, only $12.8 \%$ of the participants had experience of GBV case management within the previous two years (Table 3). The number and details of the cases are presented in Table 4. Of those who had experience in managing a GBV case, it was found that of the total number of 102 cases managed, sexual violence was the highest $(71.57 \%)$, followed by physical violence $(10.78 \%)$. Most sexual violence perpetrators were strangers, whereas most physical violence perpetrators were intimate partners.

\section{Health care practices for GBV cases and their association}

Table 5 shows the association between independent variables and health care practices concerning GBV patients in the past two years. The health care practices for GBV cases was significantly associated with education level $(\phi$-value $=0.001)$, level of hospital $(\phi$-value $=0.013)$, job position $(\phi$-value $<0.001)$ and level of knowledge $(\phi$-value $=0.029)$. Also, the GBV response training, STI management training and multidisciplinary teamwork 
JHR

35,2

Characteristics $(n=398)$

Number $(n)$

Percentage $(\%)$

Age (Years)

20-30

130

$31-40$

32.7

$41-50$

81

43.2

$51-60$

152

Mean $( \pm$ SD $)=35.01( \pm 8.265)$ Min $=20$ Max $=57$

Sex

Male

Female

Education level

Diploma

46.7

Bachelor

186

47.2

Master

Workplace

Station

Township

138

127

31.9

Regional

33.4

Job position

Junior nurse

19.8

Senior nurse

57.0

Head nurse

4.3

Medical officer

7.5

Senior medical officer

11.3

Working experience (total period since graduation)

$1-10$

55.8

$11-20$

34.9

$\geq 21$

222

9.3

Mean $( \pm \mathrm{SD})=9.91( \pm 6.82)$ Min $=1$ Max $=34$

Ever attended training on gender-based violence response

No

Yes

Ever attended training on management of STIS

No

Yes

Ever attended a training on the multidisciplinary teamwork

No

Table 1.

Yes

Ever attended training related to communication skill

training were significantly associated with health care practices at $p$-value 0.001 , $p$-value $<0.00$ and $p$-value 0.040 , respectively.

Binary logistic regression analysis of associated factors of health care practices for GBV cases

Binary logistic regression was used to find out whether there was a statistically significant relationship between the dependent variable and the independent variables which had a 


\begin{tabular}{lcr}
\hline$n=398$ & Number $(n)$ & Percentage $(\%)$ \\
\hline Level of knowledge & & 5.5 \\
Low $(<60 \%)(0-26)$ & 22 & 66.1 \\
Moderate $(60-80 \%)(27-36)$ & 263 & 28.4 \\
High $(>80 \%)(37-45)$ & 113 & \\
Mean $( \pm \mathrm{SD})=34.07( \pm 4.348)$ Min $=18$ Max $=42$ & & 17.1 \\
Level of attitude & & 73.9 \\
Negative $(0-16)$ & 68 & 9.0 \\
Neutral $(17-21)$ & 294 & 35.2 \\
Positive $(22-24)$ & & 54.3 \\
Mean $( \pm \mathrm{SD})=18.65( \pm 2.313)$ Min $=12$ Max $=24$ & 10.6 \\
Level of supportive environmental factors & & 140 \\
Poor $(1-3)$ & 216 & \\
Moderate $(4-6)$ & 42 & \\
High $(7)$ & & \\
Mean $( \pm S D)=4.29( \pm 1.643)$ Min $=0$ Max $=7$ & & \\
\end{tabular}

High $(>80 \%)(37-45)$

Neutral (17-21)

Positive (22-24)

Mean $( \pm \mathrm{SD})=18.65( \pm 2.313)$ Min $=12 \mathrm{Max}=24$

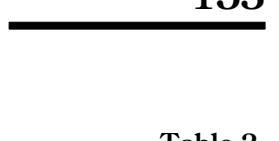

Respondents by level of knowledge, attitude and supportive environmental factors toward health care for GBV cases

\begin{tabular}{lcc}
\hline Experiences of managing a GBV case within the last two years $(n=398)$ & Number $(n)$ & Percentage $(\%)$ \\
\hline Yes & 51 & 12.8 \\
No & 347 & 87.2 \\
\hline
\end{tabular}

Table 3. Experience of GBV response and level of health care practices

\begin{tabular}{|c|c|c|c|c|}
\hline Cases $n(\%)$ & Sexual violence & Physical violence & Psychological violence & \\
\hline $\begin{array}{l}73(71.57) \\
11(10.78) \\
9(8.82) \\
4(3.92) \\
4(3.92) \\
1(0.98)\end{array}$ & $\begin{array}{l}\sqrt{ } \\
\sqrt{ } \\
\sqrt{ }\end{array}$ & $\begin{array}{l}\sqrt{ } \\
\sqrt{ } \\
\sqrt{ }\end{array}$ & $\begin{array}{l}\sqrt{ } \\
\sqrt{ }\end{array}$ & $\begin{array}{r}\text { Table } 4 . \\
\text { Details of managed } \\
\text { cases and number of } \\
\text { cases managed among } \\
\text { experienced } \\
\text { respondents }(n=102)\end{array}$ \\
\hline
\end{tabular}

$p$-value of less than 0.2 (age, education level, workplace, job position, completion of GBV response training, STI management training, multidisciplinary teamwork training and communication skills training) in bivariate analysis.

Table 6 shows the binary logistic regression analysis of each independent variable associated with health care practices for GBV cases in the past two years. From the results, the education level was found to be positively associated with health care practices for GBV cases. Health care staff with a bachelor's degree were more likely to practice than those who completed a diploma degree [OR $=3.76,95 \% \mathrm{CI}(1.85-7.65)]$.

The level of the hospital was found to be negatively associated with health care practices. Participants who worked in the regional hospitals were less likely to practice when compared with those who worked in the station hospital [OR $=0.30,95 \% \mathrm{CI}(0.13-0.70)]$. Another association factor was the job position. The medical officers had 3.889 times and senior medical officers had 3.16 times more practice experience than the junior nurses. The completion of attending the training was also associated with health care practices. Among the participants who had completed the GBV response training, the STI management 
JHR

35,2

\begin{tabular}{|c|c|c|c|}
\hline \multirow[b]{2}{*}{ Independent variables $(n=398)$} & \multicolumn{2}{|c|}{ Health care practices $n(\%)$} & \multirow[b]{2}{*}{$p$-value } \\
\hline & No & Yes & \\
\hline \multicolumn{4}{|l|}{ Socio-demographic characteristics } \\
\hline Age (Years) & & & \multirow[t]{5}{*}{0.165} \\
\hline $20-30$ & $118(90.7)$ & $12(9.3)$ & \\
\hline $31-40$ & $146(84.8)$ & $26(15.2)$ & \\
\hline $41-50$ & $72(88.8)$ & 9 (11.2) & \\
\hline $51-60$ & $11(73.3)$ & $4(26.7)$ & \\
\hline Sex & & & \multirow{3}{*}{0.514} \\
\hline Male & $46(83.6)$ & $9(16.4)$ & \\
\hline Female & $301(87.7)$ & 42 (12.3) & \\
\hline Education level & & & \multirow{4}{*}{$0.001^{*}$} \\
\hline Diploma & $175(94.1)$ & $11(5.9)$ & \\
\hline Bachelor & $152(80.9)$ & 36 (19.1) & \\
\hline Master & $20(83.3)$ & $4(16.7)$ & \\
\hline Level of hospital & & & \multirow[t]{4}{*}{$0.013^{*}$} \\
\hline Station & $114(82.6)$ & $24(17.4)$ & \\
\hline Township & $108(85.0)$ & 19 (15.0) & \\
\hline Regional & $125(94.0)$ & $8(6.0)$ & \\
\hline Job position & & & \multirow[t]{6}{*}{$\#<0.001^{*}$} \\
\hline Junior nurse & 70 (88.6) & 9 (11.4) & \\
\hline Senior nurse & 208 (91.6) & $19(8.4)$ & \\
\hline Head nurse & $17(100.0)$ & $0(0.0)$ & \\
\hline Medical officer & $20(66.6)$ & $10(33.4)$ & \\
\hline Senior medical officer & $32(71.1)$ & $13(28.9)$ & \\
\hline Working experience (total years) & & & \multirow[t]{5}{*}{$\# 0.762$} \\
\hline $1-10$ & $194(87.4)$ & $28(12.6)$ & \\
\hline $11-20$ & $122(87.8)$ & 17 (12.2) & \\
\hline $21-30$ & 29 (82.9) & $6(17.1)$ & \\
\hline $31-40$ & $2(100.0)$ & $0(0.0)$ & \\
\hline$G B V$ response training & & & \multirow[t]{2}{*}{$\# 0.001^{*}$} \\
\hline No & $334(88.8)$ & $42(11.2)$ & \\
\hline Yes & $13(59.1)$ & $9(40.9)$ & \multirow{4}{*}{$<0.001^{*}$} \\
\hline STI management training & & & \\
\hline No & 238 (91.9) & $21(8.1)$ & \\
\hline Yes & 109 (78.4) & $30(21.6)$ & \\
\hline Multidisciplinary teamwork training & & & \multirow[t]{3}{*}{$\# 0.040^{*}$} \\
\hline No & $325(88.3)$ & 43 (11.7) & \\
\hline Yes & $22(73.3)$ & $8(26.7)$ & \\
\hline Communication skills training & & & \multirow{3}{*}{0.193} \\
\hline No & $280(88.3)$ & 37 (11.7) & \\
\hline Yes & $67(82.7)$ & 14 (17.3) & \\
\hline Level of knowledge & & & \multirow[t]{4}{*}{$0.029 *$} \\
\hline Low & $22(100.0)$ & $0(0.0)$ & \\
\hline Moderate & $233(88.6)$ & 30 (11.4) & \\
\hline High & $92(81.4)$ & 21 (18.6) & \\
\hline Level of attitude & & & \multirow{4}{*}{0.907} \\
\hline Low & $60(88.2)$ & $8(11.8)$ & \\
\hline Moderate & $255(86.7)$ & 39 (13.3) & \\
\hline High & $32(88.8)$ & $4(11.2)$ & \\
\hline Level of supportive environmental factors & & & \multirow[t]{4}{*}{0.562} \\
\hline Poor & $125(89.3)$ & $15(10.7)$ & \\
\hline Moderate & $187(86.6)$ & 29 (13.4) & \\
\hline High & 35 (83.3) & 7 (16.7) & \\
\hline Note(s): \#Fisher's exact test, ${ }^{*} p$-value & & & \\
\hline
\end{tabular}

Table 5.

Association between independent variables and the health care practices

\section{4}

154

Socio-demographic characteristics Age (Years)

$31-40$

$41-50$

Sex

Education level

Diploma

Bachelor

Station

Township

Regional

Job position

Junior nurse

ior nurse

Medical officer

Senior medical officer

Working experience (total years)

$11-20$

21-30

31-40

GBV response training

Yes

STI management training

Multidisciplinary teamwork training

Communication skills training

Level of knowledge

Low

High

Level of attitude

Moderate

High

Poor

Moderate

Note(s): \#Fisher's exact test, ${ }^{*} p$-value $<0.05$
Health care practices $n(\%)$

$146(84.8)$

$72(88.8)$

11 (73.3)

46 (83.6)

301 (87.7)

175 (94.1)

152 (80.9)

20 (83.3)

114 (82.6)

108 (85.0)

$25(94.0)$

$208(916)$

$17(100.0)$

20 (66.6)

32 (71.1)

94 (87.4)

(82.9)

334 (88.8)

13 (59.1)

238 (91.9)

109 (78.4)

325 (88.3)

22 (73.3)

280 (88.3)

233 (88.6)

55 (86.7)

$125(89.3)$

187 (86.6)

7 (16.7) 


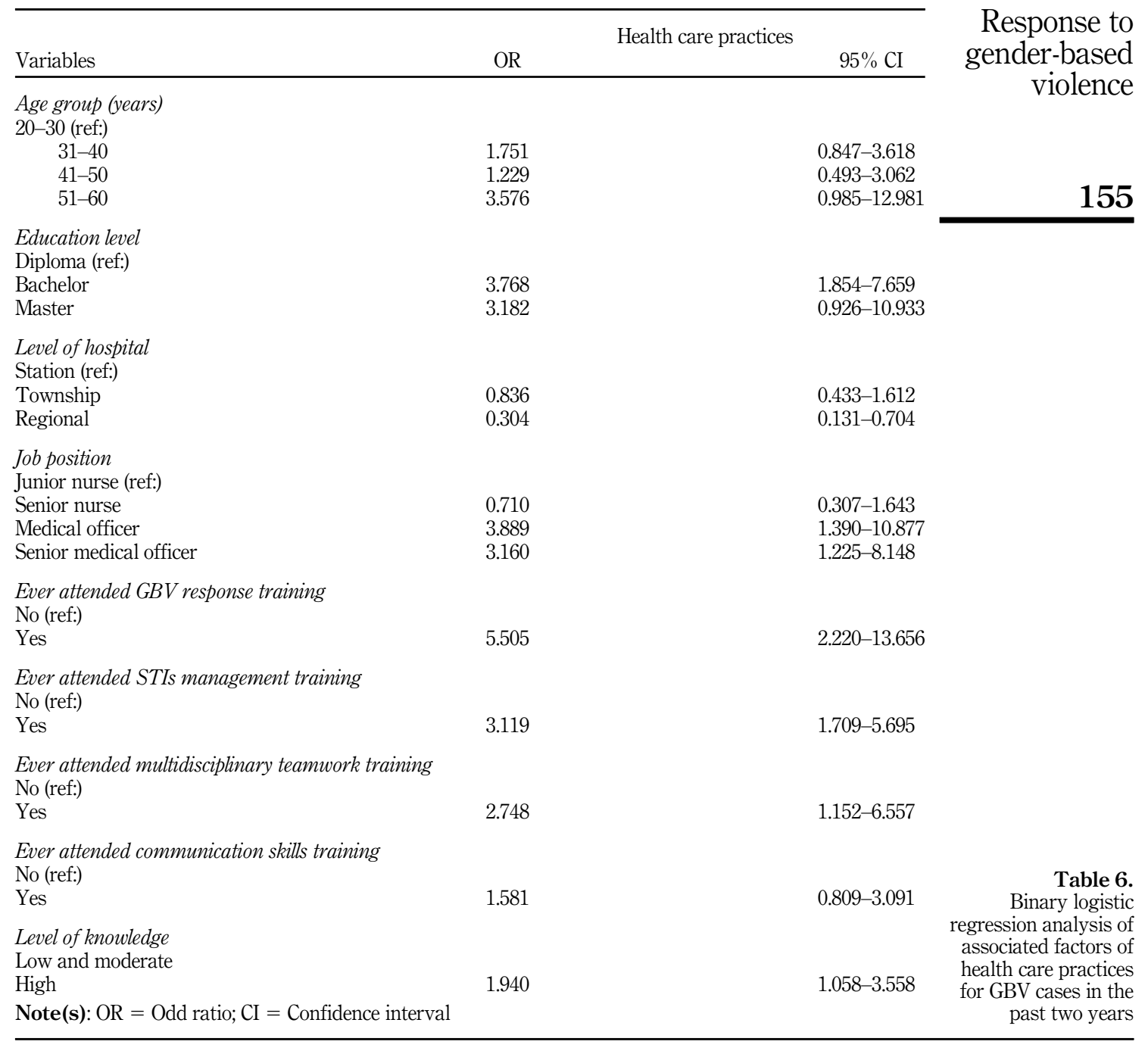

training and the multidisciplinary teamwork training, the odds of practice were 5.5, 3.1 and 2.7 times higher, respectively, when compared to those who had not completed the training. The health care personnel who had high levels of knowledge were 1.94 times more likely to practice when compared to those who had a low level of knowledge.

\section{Discussion}

The principal finding of the health care practices for GBV cases among 398 health care personnel indicated that only $12.8 \%(n=51)$ had ever managed GBV cases. Most cases were sexual violence cases. This is because the Myanmar community sees only rape cases as GBV and requiring medical attention [18]. 
JHR

35,2

Among them $(n=51)$, the mean score of practice was $12.90 \pm 7.45(\max =34$, $\min =0)$, which indicates that the scores for the practice are quite low and possibly did not practice well in the real situation. This finding matches the study conducted by the Gender Equality Network, which stated that in the Myanmar health system, there is no established GBV response program and the services are more focused on forensic medicine than the GBV specific response [18, 19].

In this study, the majority of the respondents were aged between $31-40$ years old ( $86.2 \%)$, and around $47 \%$ accounted for either a diploma or a bachelor's degree. Participants were selected from various levels of hospitals (station, township and regional) in Yangon Region, Myanmar. The vast majority of them were senior nurses, and other positions included were junior nurses, head nurses, medical officers and senior medical officers. More than half of the respondents had worked for 1-10 years, and the working experience ranged from 1 to 34 years.

Interestingly, $95 \%$ of the respondents had not attended the GBV response training despite the Ministry of Health and Sports launching the Guideline on Health Care Response for GBV Survivors and Training of Trainers in October 2018 in Naypyidaw [19]. The participants of these events were from the administrative levels from each region in Myanmar. The training was aimed to be implemented at all levels of all hospital types. The Yangon Regional Health Department provided GBV response training on 19-25 June 2019 with the coordination of the Gender-Equality Network, a local NGO, after completion of this current study [19].

In bivariate analysis, age, education level, workplace, job position, GBV response training, STI management training, multidisciplinary teamwork training and communication skills training were found to be associated with health care practices. Next, in the multivariate analysis, the education level was found to be associated with the level of health care practices. The higher the education level of the participants, the better the health care practice they had. Similarly, health care personnel who had completed the GBV response training and STIs management and multidisciplinary teamwork training had more practice than those who had not attended either training program. Our result was consistent with another quasiexperimental study - the practice of the health care workers on post-rape care - that was also found to have increased by about $50 \%$ after the intervention training [20]. The positive association between STI management training and health care practices was also found in a similar study conducted in four humanitarian settings (Kenya, Jordan, Ethiopia and DRC) in 2010-2012 [21].

Regarding knowledge, only $28.4 \%$ of the respondents had a high knowledge level. The results indicate that the participants should be educated and trained in every aspect of knowledge toward both understanding GBV cases and the need for a proactive health care response.

From the bivariate analysis, the level of knowledge was found to be statistically associated with the health care practice. Participants with a higher knowledge tended to have more experience of practice. In a training assessment in Kabul, Afghanistan, the WHO found out that after the training, with increasing knowledge on GBV, the participants were found to practice the identification, counseling, examination, treatment and referral of GBV cases more often [22].

Generally, most of the participants had a neutral attitude toward health care for GBV cases; $66.6 \%$ of the respondents agreed that health care personnel were responsible for screening suspected GBV cases. More than half of the participants $(56.3 \%)$ disagreed that domestic violence is a private matter, whereas $70 \%$ of respondents from a study on Intimate Partner Violence (IPV) carried out in Turkey in 2007 stated that this did not interfere with private family matters [23]. So, the average respondents in this study knew that domestic violence is a public issue, and it is also a human right violation as described in the GBV guideline distributed by the Myanmar Ministry of Health and Sports (MOHS). 
From the bivariate analysis, even in this study, the level of attitude was not statistically associated with the health care practice. However, other studies show that health care providers with a positive attitude have better practices toward domestic violence response $[24,25]$.

\section{Conclusion and recommendation}

In this study, age, education level, workplace, job position, completion of GBV response training, STI management training, multidisciplinary teamwork training, communication skills training and level of knowledge were found to be associated with the binary logistic regression analysis. But after processing the multivariate analysis, only education level, job position, GBV response training, STI management training, multidisciplinary teamwork training and level of knowledge were statistically, significantly and positively associated with the level of health care practices.

However, this study cannot be generalized to the whole population. Besides, as most participants identified GBV as a rape case, the number of GBV cases they have encountered in the past two years cannot be exactly correct, and recall bias can also have occurred. Very few, but a significant number of participants didn't even know what GBV is and this can also be a cofounder. Social desirability bias can also have occurred in the practical part of this research even though the researcher explained that the responses were confidential.

The findings from this study helped advocate and guide the public health manager in strengthening the readiness for GBV response at the service provider level. The results indicated that the general knowledge on both GBV and health facility response for GBV of the health service providers should be improved and the readiness of the health care facility should be further strengthened. As many GBV victims usually seek help from health care services, early detection of violence by the health care providers can reduce or eliminate the negative impacts of GBV and prevent further incidents [15, 25]. From the current finding, the $\mathrm{MOPH}$ used the results to recruit participants in GBV response training covering management level and service provider level, with details of the level of hospitals being taken into account as well.

Next, the awareness of GBV can be enhanced among the health care providers who can act as awareness promoters and thus spread information on accessible services for GBV clients to community members and thereby encourage community participation in GBV response and prevention $[7,17]$. The final hope is to prevent GBV by sharing information.

Future studies should focus on all aspects of knowledge and practice toward GBV response. Furthermore, as training on GBV response was given to the health care providers in the Yangon Region following this study, a post-intervention study is recommended. Ideally, any further studies could explore more options to enhance data gathering such as interviews, workshops and focused group discussions as well as more diverse participants including medical-social workers, health assistants, midwives and female health visitors. Finally, the Ministry of Health and Sports should also establish specific programs for GBV response and should promote health care practices regarding health sector response for GBV cases.

\section{References}

1. United Nations, Office of the united nations high commissioner for human rights [OHCHR]. Declaration on the elimination of violence against women. Geneva: OHCHR; 1994.

2. World Health Organization [WHO], London School of Hygiene and Tropical Medicine, South African Medical Research Council. Global and regional estimates of violence against women: prevalence and health effects of intimate partner violence and non-partner sexual violence. Geneva: WHO; 2013. 
JHR

35,2

3. Educational Initiatives, Asia Justice and Rights. Violence against women (VAW) prevention and response services and access in Myanmar. [cited 2019 Aug]. Available at: http://www.asia-ajar. org/files/VAW\%20Preveition\%20Myanmar.pdf.

4. United Nations Entity for Gender Equality and the Empowerment of Women [UN Women]. ASEAN regional guidelines on violence against women and girls data collection and use. Bangkok: UN Women; 2018.

5. Boyd C. The impacts of sexual assault on women (ACSSA Resource Sheet). Melbourne: Australian Institute of Family Studies; 2011.

6. UNFPA Asia and the Pacific Regional Office. Health sector response to gender-based violence: an assessment of the Asia Pacific region. Bangkok: UNFPA; 2010.

7. The United Nations High Commissioner for Refugees [UNHCR]. Prevention and response to sexual and gender-based violence in refugee situations. Geneva: UNHCR; 2001.

8. World Health Organization [WHO]. Clinical management of rape survivors: developing protocols for use with refugees and internally displaced persons. Geneva: WHO; 2004.

9. Myanmar, Ministry of Health and Sports. Guideline on health care response for gender-based violence (GBV) survivors. Nay Pyi Taw: Ministry; 2018.

10. Greifinger R, Richards LM, Oo S, Khaing EE, Thet MM. The role of the private health sector in responding to gender-based violence in Myanmar. [cited 2019 Aug]. Available at: https://www.psi. org/wp-content/uploads/2015/10/Richards-Myanmar-FINAL.pdf.

11. Chew KS, Noredelina MN, Ida ZZ. Knowledge, attitude and practice among healthcare staffs in the emergency department, hospital universiti sains Malaysia towards rape victims in one Stop Crisis Centre (OSCC). Med J Malaysia. 2015 Jun; 70(3): 162-8.

12. Jina R, Jewkes R, Christofides N, Loots L. Knowledge and confidence of South African health care providers regarding post-rape care: a cross-sectional study. BMC health services research. 2013 Jul; 13: 257. doi: 10.1186/1472-6963-13-257.

13. Cho OH, Cha KS, Yoo YS. Awareness and attitudes towards violence and abuse among emergency nurses. Asian Nurs Res (Korean Soc Nurs Sci) 2015 Sep; 9(3): 213-8. doi: 10.1016/j.anr.2015.03.003.

14. Shahali S, Mohammadi E, Lamyian M, Kashanian M, Eslami M, Montazeri A. Barriers to healthcare provision for victims of sexual assault: a grounded theory study. Iran Red Crescent Med J. 2016 Mar; 18(3): e21938. doi: 10.5812/ircmj.21938.

15. Swedish International Development Cooperation Agency [SIDA]. Preventing and responding to gender-based violence: expression and strategies. Stockholm: SIDA; 2015.

16. U.S. Agency for International Development [USAID], The RESPOND Project. Baseline assessment of the readiness of health facilities to respond to gender-based violence in Guinea. New York: The RESPOND Project; 2012.

17. Bott S, Guedes A, Claramunt MC, Guezmes A. Improving the health sector response to gender based violence: a resource manual for health care professionals in developing countries. New York: International Planned Parenthood Federation; 2010.

18. Myanmar, The Gender Equality Network [GEN]. Service provision for gender-based violence survivors in Myanmar. Yangon: GEN; 2018.

19. Myanmar, Ministry of Health and Sport. News. 2019. [cited 2019 Aug]. Available at: https://www. mohs.gov.mm.

20. Abeid M, Muganyizi P, Mpembeni R, Darj E, Axemo P. Evaluation of a training program for health care workers to improve the quality of care for rape survivors: a quasi-experimental design study in Morogoro, Tanzania. Glob Health Action. 2016; 9: 31735. doi: 10.3402/gha.v9.31735.

21. Smith JR, Ho LS, Langston A, Mankani N, Shivshanker A, Perera D. Clinical care for sexual assault survivors multimedia training: a mixed-methods study of effect on healthcare providers' attitudes, knowledge, confidence, and practice in humanitarian settings. Confl Health. 2013; 7(1): 14. doi: $10.1186 / 1752-1505-7-14$. 
22. Word Health Organization [WHO]. WHO project improves health care sector's response to genderbased violence in Afghanistan. Afghanistan: WHO; 2018.

23. Aksan HA, Aksu F. The training needs of Turkish emergency department personnel regarding intimate partner violence. BMC Publ Health. 2007 Dec; 7(1): 350. doi: 10.1186/1471-2458-7-350.

Response to gender-based violence

24. Yousefnia N, Nekuei N, Farajzadegan Z. The relationship between healthcare providers' performance regarding women experiencing domestic violence and their demographic characteristics and attitude towards their management. J Inj Violence Res. 2018 Jul; 10(2): 113-8. doi: 10.5249/jivr.v10i2.958.

25. Garcia-Moreno C, Jansen HA, Ellsberg M, Heise L, Watts CH. Prevalence of intimate partner violence: findings from the WHO multi-country study on women's health and domestic violence. Lancet. 2006 Oct; 368(9543): 1260-9. doi: 10.1016/s0140-6736(06)69523-8.

\section{Corresponding author}

Montakarn Chuemchit can be contacted at: montakarn.ch@chula.ac.th

For instructions on how to order reprints of this article, please visit our website:

www.emeraldgrouppublishing.com/licensing/reprints.htm

Or contact us for further details: permissions@emeraldinsight.com 